\title{
Stabilisation of stochastic differential equations with Markovian switching by feedback control based on discrete-time state observation with a time delay
}

\author{
Qinwei Qiu ${ }^{\mathrm{a}}$, Wei Liu ${ }^{\mathrm{b}, *}$, Liangjian $\mathrm{Hu}^{\mathrm{c}}$, Xuerong $\mathrm{Mao}^{\mathrm{d}}$, Surong $\mathrm{You}^{\mathrm{c}}$ \\ ${ }^{a}$ College of Information Sciences and Technology, Donghua University, Shanghai, 201620, China \\ ${ }^{b}$ Department of Mathematics, Shanghai Normal University, Shanghai, 200234, China \\ ${ }^{c}$ Department of Applied Mathematics, Donghua Univerisity, Shanghai 201620, China \\ ${ }^{d}$ Department of Mathematics and Statistics, University of Strathclyde, Glasgow G1 1XH, U.K.
}

\begin{abstract}
Feedback control based on discrete-time state observation for stochastic differential equations with Markovian switching was initialled by Mao (2013). In practice, various effects could cause some time delay in the control function. Therefore, the time delay is taken into account for the discrete-time state observation in this paper and the mean-square exponential stability of the controlled system IS investigated. This paper is devoted as a continuous research to Mao (2013).

Keywords: Mean-square exponential stability, Discrete-time state observation, Time delay, Stochastic differential equations with Markovian switching
\end{abstract}

\section{Introduction}

Stochastic differential equations (SDEs) with Markovian switching in recent years have been attracting a lot of attention. We refer the readers to monographs $[1,2]$ on this field for the detailed introduction. As the states of parameters of SDEs can be changed according to some Markov processes, SDEs with Markovian switching are powerful tools to describe systems which encounter abrupt changes in the structure $[? 5,4,3,6]$.

One important aspect of the study on SDEs with Markovian switching is the automatic control. There are many papers devoted to this field with emphasis on the asymptotic analysis of stability, and we just mention some of the works $[14,15,8,13,19,17,18,20,21,22,23,24]$ and the references therein. One classical problem of this field is that for some given unstable SDE system

$$
d(x(t))=f(x(t), r(t), t) d t+g(x(t), r(t), t) d w(t),
$$

how one could design a control function $u(x(t), r(t), t)$ embedded into the drift coefficient such that the modified system

$$
d(x(t))=(f(x(t), r(t), t)+u(x(t), r(t), t)) d t+g(x(t), r(t), t) d w(t)
$$

\footnotetext{
* Corresponding author

Email address: weiliu@shnu.edu.cn;lwbvb@hotmail.com (Wei Liu)
} 
is stable.

However, in practice one may argue that it is expensive and impractical to design the control function $u(x(t), r(t), t)$ based on the continuous state of $x(t)$. To tackle this drawback, Mao in [16] developed the technique of feedback control based on discrete-time state observation, and that work is the first paper applying this idea to SDEs with Markovian switching. It should be noted that the deterministic counterparts have been widely discussed, such as $[7,9,10,11,12]$.

The idea in [16] is that instead of using the continuous state of $x(t)$ to construct the feedback control function $u(x(t), r(t), t)$ the author only needs a sequence of countable state $x(\lfloor t / \tau\rfloor \tau)$, where $\tau>0$ is a constant and $\lfloor t / \tau\rfloor$ is the integer part of $t / \tau$. The control function is then designed as $u(x(\lfloor t / \tau\rfloor \tau), r(t), t)$ and one can see only the states at $t=0, \tau, 2 \tau, \ldots$ are needed. The resulting controlled system is

$$
d x(t)=(f(x(t), r(t), t)+u(x(\lfloor t / \tau\rfloor \tau), r(t), t)) d t+g(x(t), r(t), t) d w(t) .
$$

Applying this new technique, one can design the control function with relatively low cost. Due to the general techniques used in proofs in [16], the $\tau$ was required to be quite small in that paper. Mao and his group, therefore, modified the techniques and released the restriction on $\tau$ in $[26,27]$. We further observe that various effects, such as data transmission, may be time consuming. Therefore, some time delay may occur in the control function $u(x(\lfloor t / \tau\rfloor \tau), r(t), t)$. Based on this observation, we propose some time delay when constructing the feedback control based on discrete-time state observation in this paper. And the new controlled system briefly reads as (please see the details in Section 2)

$$
d x(t)=(f(x(t), r(t), t)+u(x(\delta(t)), r(t), t)) d t+g(x(t), r(t), t) d w(t)
$$

where

$$
\delta(t)=\lfloor t / \tau\rfloor \tau-\tau_{0},
$$

$\tau_{0}$ is the delay time and $\tau$ is the discrete-time observation gap.

One may notice that in (1.1) the time delay has already occurred in the control function $u(x(\lfloor t / \tau\rfloor \tau), r(t), t)$ when $k \tau<t<(k+1) \tau$ for any positive integer $k$. But when $t=k \tau$, the time delay in the control function $u(x(\lfloor t / \tau\rfloor \tau), r(t), t)$ is dismissed. Actually, the appearance of the time delay in (1.1) is not deliberate but due to the discrete-time state observation. On the contrary, the time delay in this paper is designed on purpose. Thus the proofs in this work are different from those in [16]. It should also be mentioned that the feedback control based on continuous state observation with a time delay was discussed in [25]. This paper is constructed in the following way. In Section 2 some mathematical preliminaries are given. Section 3 sees the main results of the linear case, and Section 4 is devoted to the nonlinear case. Some brief examples on the design of the control functions and numerical simulations are displayed in Section 5. Section 6 concludes this paper with some possible future research. 


\section{Mathematical Preliminaries}

Throughout this paper, let $\left(\Omega, \mathcal{F},\left\{\mathcal{F}_{t}\right\}_{t \geq 0}, \mathbb{P}\right)$ be a complete probability space with a filtration $\left\{\mathcal{F}_{t}\right\}_{t \geq 0}$ satisfying the usual conditions that it is right continuous and $\mathcal{F}_{0}$ contains all $\mathbb{P}$-null sets. Let $w(t)=\left(w_{1}(t), \cdots, w_{m}(t)\right)^{T}$ be an $m$-dimensional Brownian motion defined on the probability space. For a vector or matrix $A$, its transpose is denoted by $A^{T}$. For $x \in R^{n}$, its Euclidean norm is denoted by $|x|$. The trace and operator norms of a matrix $A$ are denoted by $|A|=\sqrt{\operatorname{trace}\left(A^{T} A\right)}$ and $\|A\|=\max \{|A x|:|x|=1\}$, respectively. For a symmetric matrix $A$ i.e. $A=A^{T}$, its smallest and largest eigenvalues are denoted by $\lambda_{\min }(A)$ and $\lambda_{\max }(A)$, respectively. By $A \leq 0$ and $A<0$, we mean $A$ is non-positive and negative definite, respectively. Denote by $L_{\mathcal{F}_{t}}^{2}\left(R^{n}\right)$ the family of all $\mathcal{F}_{t}$-measurable $R^{n}$-valued random variables $\xi$ such that $\mathbb{E}|\xi|^{2}<\infty$, where $\mathbb{E}$ is the expectation with respect to the probability measure $\mathbb{P}$. For a real number $a$, let $\lfloor a\rfloor$ denote the integer part of $a$ and $\lceil a\rceil$ denote the smallest integer larger than $a$. If both $a$ and $b$ are real numbers, then $a \vee b=\max \{a, b\}$ and $a \wedge b=\min \{a, b\}$.

Let $r(t), t \geq 0$, be a right-continuous Markov chain on the probability space taking values in a finite state space $S=\{1,2, \cdots, N\}$ with generator $\Gamma=\left(\gamma_{i j}\right)_{N \times N}$ given by

$$
\mathbb{P}\{r(t+\Delta)=j \mid r(t)=i\}= \begin{cases}\gamma_{i j} \Delta+o(\Delta) & \text { if } i \neq j, \\ 1+\gamma_{i i} \Delta+o(\Delta) & \text { if } i=j,\end{cases}
$$

where $\Delta>0$. Here $\gamma_{i j} \geq 0$ is the transition rate from $i$ to $j$ if $i \neq j$ while

$$
\gamma_{i i}=-\sum_{j \neq i} \gamma_{i j}
$$

We assume that the Markov chain $r(\cdot)$ is independent of the Brownian motion $w(\cdot)$. It is known that almost all sample paths of $r(t)$ are constant except for a finite number of simple jumps in any finite subinterval of $R_{+}(:=[0, \infty))$. We stress that almost all sample paths of $r(t)$ are right continuous.

Now we demonstrate the derivation of the SDE with Markovian switching that we will investigate.

Consider an $n$-dimensional unstable hybrid SDE

$$
d y(t)=f(y(t), r(t), t) d t+g(y(t), r(t), t) d \omega(t), \quad \text { for } t \geq 0
$$

with initial data $y(0)=y_{0} \in L_{\mathcal{F}_{0}}^{2}\left(\mathbb{R}^{n}\right)$ and $r(0)=r_{0}$. The drift and diffusion coefficients $f: \mathbb{R}^{n} \rightarrow \mathbb{R}^{n}$ and $g: \mathbb{R}^{n} \rightarrow \mathbb{R}^{n \times m}$ satisfy the global Lipschitz condition that for any $x_{1}, x_{2} \in \mathbb{R}^{n}$

$$
\left|f\left(x_{1}\right)-f\left(x_{2}\right)\right|^{2} \vee\left|g\left(x_{1}\right)-g\left(x_{2}\right)\right|^{2} \leq K\left|x_{1}-x_{2}\right|^{2},
$$

where $K$ is a positive constant.

For some time delay $\tau_{0}>0$ and the discrete-time observation time mesh $\tau>0$, one could design the control function and the controlled system reads as

$$
d x(t)=(f(x(t), r(t), t)+u(x(\delta(t)), r(t), t)) d t+g(x(t), r(t), t) d w(t),
$$


where

$$
\delta(t)=\lfloor t / \tau\rfloor \tau-\tau_{0} .
$$

One may notice that original system (2.1) only provides initial data at $t=0$, but the new system (2.2) requires initial data $x_{\theta}:=\left\{x(\theta),-\tau_{0} \leq \theta \leq 0\right\}$. To tackle this problem, the original system (2.1) is allowed to evolve for a period of $\tau_{0}$ then the state observations during this period are regarded as the initial data for the new system $(2.2)$, that is

$$
y(t)=x\left(t-\tau_{0}\right) \text {, for } 0 \leq t \leq \tau_{0} .
$$

By [1], we know that under the global Lipschitz condition on $f$ and $g$ the original system (2.1) does not explode during $\left[0, \tau_{0}\right]$, i.e.

$$
\sup _{-\tau_{0} \leq t \leq 0} \mathbb{E}|x(t)|^{2}=\sup _{0 \leq t \leq \tau_{0}} \mathbb{E}|y(t)|^{2} \leq C,
$$

where $C$ is a positive constant.

In the proofs in next two sections, due to the techniques applied we need some extra data $x_{\theta}=$ $\left\{x(\theta),-2 \tau_{0}-\tau \leq \theta<-\tau_{0}\right\}$ for the system (2.2). To deal with this issue, we simply set $x(\theta)=y(0)$ for all $\theta \in\left[-2 \tau_{0}-\tau, \tau_{0}\right)$. This setting is reasonable as in the real life simulation we still only need $x_{\theta}:=\left\{x(\theta),-\tau_{0} \leq \theta \leq 0\right\}$.

\section{Main Result: Linear Case}

In this section, we study the stability theory of the following linear hybrid system

$$
d x(t)=[A(r(t))+u(x(\delta(t)), r(t), t)] d t+\sum_{k=1}^{m} B_{k}(r(t)) x(t) d \omega_{k}(t) \quad t \geq 0
$$

with the initial data $x_{\theta}:=\left\{x(\theta),-2 \tau_{0}-\tau \leq \theta \leq 0\right\}$, where $A, B_{k}: S \rightarrow R^{n}$ and we will often write $A(i)=A_{i}$ and $B_{k}(i)=B_{k, i}$.

Since the original system is linear, we consider the linear feedback control here. One of the most commonly used linear feedback control is the structure control of the form $u(x, i)=F(i) G(i) x$, where $F(i): S \rightarrow \mathbb{R}^{n \times l}$ and $G(i): S \rightarrow \mathbb{R}^{l \times n}$. We design the control function as $u(x(\delta(t)), r(t), t)=$ $F(r(t)) G(r(t)) x(\delta(t))$, which is based on the discrete-time state observations. There are two cases that

- State feedback: design $F(\cdot)$ when $G(\cdot)$ is given.

- Output injection: design $G(\cdot)$ when $F(\cdot)$ is given.

Let us introduce an useful lemma which is important in the proof of the main theory.

lemma $x-y \quad$ Lemma 3.1. Let

$$
M_{A}=\max _{i \in S}\left\|A_{i}\right\|^{2}, \quad M_{F G}=\max _{i \in S}\left\|F_{i} G_{i}\right\|^{2}, \quad M_{B}=\max _{i \in S} \sum_{k=1}^{m}\left\|B_{k, i}\right\|^{2},
$$


and

$$
K\left(\tau, \tau_{0}\right)=\tau\left[6\left(\tau+\tau_{0}\right) M_{A}+6 M_{B}+3\left(\tau+\tau_{0}\right) M_{F G}\right] e^{\left[6\left(\tau+\tau_{0}\right) M_{A}+6 M_{B}\right]\left(\tau+\tau_{0}\right)}
$$

If both $\tau>0$ and $\tau_{0}>0$ are sufficiently small such that for $2(h+1) K\left(\tau, \tau_{0}\right)<1$ where $h=\left\lceil\tau_{0} / \tau\right\rceil$, then the solution of (3.1) satisfies

$$
\begin{aligned}
\int_{0}^{t} \mathbb{E}|x(s)-x(\delta(s))|^{2} d s & \leq \frac{2(h+1) K\left(\tau, \tau_{0}\right)}{1-2(h+1) K\left(\tau, \tau_{0}\right)} \int_{0}^{t} \mathbb{E}|x(s)|^{2} d s \\
& +\frac{\tau\left(h+h^{2}\right) K\left(\tau, \tau_{0}\right)}{2-4(h+1) K\left(\tau, \tau_{0}\right)} \sup _{\theta \in\left[-\tau_{0}, 0\right]} \mathbb{E}|x(\theta)|^{2} .
\end{aligned}
$$

for any $t \geq 0$.

Proof. Because of the definition of $x(t), x(\delta(t))$ have different forms when $0 \leq t<h \tau$ and $t \geq h \tau$, thus we separate our proof into two parts. Firstly, let $n \geq h$ be an integer, for any $t \in[n \tau,(n+1) \tau)$ we have $\delta(t)=n \tau-\tau_{0} \geq 0$. It follows from (3.1) that

$$
\begin{aligned}
x(t)-x(\delta(t))= & \int_{n \tau-\tau_{0}}^{t}[A(r(s)) x(s)+F(r(s)) G(r(s)) x(\delta(s))] d s \\
& +\sum_{k=1}^{m} \int_{n \tau-\tau_{0}}^{t} B_{k}(r(s)) x(s) d \omega_{k}(s) .
\end{aligned}
$$

Taking square and expectation on both sides, by the elementary inequality that $\left|\sum_{i=1}^{m} a_{i}\right|^{2} \leq m \sum_{i=1}^{m}\left|a_{i}\right|^{2}$ we have

$$
\begin{aligned}
& \mathbb{E}|x(t)-x(\delta(t))|^{2} \\
\leq & 3\left(\mathbb{E}\left|\int_{n \tau-\tau_{0}}^{t} A(r(s)) x(s) d s\right|^{2}+\mathbb{E}\left|\int_{n \tau-\tau_{0}}^{t} F(r(s)) G(r(s)) x(\delta(s)) d s\right|^{2}\right. \\
+ & \left.\mathbb{E}\left|\sum_{k=1}^{m} \int_{n \tau-\tau_{0}}^{t} B_{k}(r(s)) x(s) d \omega_{k}(s)\right|^{2}\right) .
\end{aligned}
$$

By the Hölder inequality, the Itô isometry and the elementary inequality, we have

$$
\begin{aligned}
& \mathbb{E}|x(t)-x(\delta(t))|^{2} \\
\leq & 3\left[\left(\tau+\tau_{0}\right) M_{A}+M_{B}\right] \int_{n \tau-\tau_{0}}^{t} \mathbb{E}|x(s)|^{2} d s+3\left(\tau+\tau_{0}\right) M_{F G} \int_{n \tau-\tau_{0}}^{t} \mathbb{E}|x(\delta(s))|^{2} d s \\
\leq & {\left[6\left(\tau+\tau_{0}\right) M_{A}+6 M_{B}\right] \int_{n \tau-\tau_{0}}^{t} \mathbb{E}|x(s)-x(\delta(s))|^{2} d s } \\
+ & {\left[6\left(\tau+\tau_{0}\right) M_{A}+6 M_{B}+3\left(\tau+\tau_{0}\right) M_{F G}\right] \int_{n \tau-\tau_{0}}^{t} \mathbb{E}|x(\delta(s))|^{2} d s }
\end{aligned}
$$

Set $h=\left\lceil\tau_{0} / \tau\right\rceil$, by dividing the interval $\left[n \tau-\tau_{0}, t\right]$ into $h+1$ subintervals we have

$$
\begin{aligned}
& \mathbb{E}|x(t)-x(\delta(t))|^{2} \\
\leq & {\left[6\left(\tau+\tau_{0}\right) M_{A}+6 M_{B}\right] \int_{n \tau-\tau_{0}}^{t} \mathbb{E}|x(s)-x(\delta(s))|^{2} d s } \\
+ & {\left[6\left(\tau+\tau_{0}\right) M_{A}+6 M_{B}+3\left(\tau+\tau_{0}\right) M_{F G}\right] \sum_{k=0}^{h} \int_{(n-k) \tau}^{(n-k+1) \tau} \mathbb{E}|x(\delta(s))|^{2} d s . }
\end{aligned}
$$


Since $\delta(s)$ is a constant when $s$ is in each subinterval, we have

$$
\begin{aligned}
& \mathbb{E}|x(t)-x(\delta(t))|^{2} \\
\leq & {\left[6\left(\tau+\tau_{0}\right) M_{A}+6 M_{B}\right] \int_{n \tau-\tau_{0}}^{t} \mathbb{E}|x(s)-x(\delta(s))|^{2} d s } \\
+ & \tau\left[6\left(\tau+\tau_{0}\right) M_{A}+6 M_{B}+3\left(\tau+\tau_{0}\right) M_{F G}\right] \sum_{k=0}^{h} \mathbb{E}\left|x\left((n-k) \tau-\tau_{0}\right)\right|^{2} .
\end{aligned}
$$

By the Gronwall inequality, we obtain that for any integer $n \geq 0$ and $t \in[n \tau,(n+1) \tau)$

$$
\mathbb{E}|x(t)-x(\delta(t))|^{2} \leq K\left(\tau, \tau_{0}\right) \sum_{k=0}^{h} \mathbb{E}\left|x\left((n-k) \tau-\tau_{0}\right)\right|^{2} .
$$

So far, (3.5) holds for $t \geq h \tau$.

Secondly, let $0 \leq n \leq h-1$ be an integer, for any $t \in[n \tau,(n+1) \tau)$ we have $\delta(t)=n \tau-\tau_{0}<0$, then we have

$$
\begin{aligned}
& x(t)-x(\delta(t))=x(t)-x(0)+x(0)-x(\delta(t)) \\
= & \int_{0}^{t}[A(r(s)) x(s)+F(r(s)) G(r(s)) x(\delta(s))] d s+\sum_{k=1}^{m} \int_{0}^{t} B_{k}(r(s)) x(s) d \omega_{k}(s) \\
+ & \int_{n \tau-\tau_{0}}^{0} A(r(s)) x(s) d s+\sum_{k=1}^{m} \int_{n \tau-\tau_{0}}^{0} B_{k}(r(s)) x(s) d \omega_{k}(s) \\
= & \int_{n \tau-\tau_{0}}^{t} A(r(s)) x(s) d s+\sum_{k=1}^{m} \int_{n \tau-\tau_{0}}^{t} B_{k}(r(s)) x(s) d \omega_{k}(s) \\
+ & \int_{0}^{t} F(r(s)) G(r(s)) x(\delta(s)) d s
\end{aligned}
$$

Using the same technique in the first part, we can get

$$
\begin{aligned}
& \mathbb{E}|x(t)-x(\delta(t))|^{2} \\
\leq & 3\left(\mathbb{E}\left|\int_{n \tau-\tau_{0}}^{t} A(r(s)) x(s) d s\right|^{2}+\mathbb{E}\left|\int_{0}^{t} F(r(s)) G(r(s)) x(\delta(s)) d s\right|^{2}\right. \\
+ & \left.\mathbb{E}\left|\sum_{k=1}^{m} \int_{n \tau-\tau_{0}}^{t} B_{k}(r(s)) x(s) d \omega_{k}(s)\right|^{2}\right) \\
\leq & 3\left[\left(\tau+\tau_{0}\right) M_{A}+M_{B}\right] \int_{n \tau-\tau_{0}}^{t} \mathbb{E}|x(s)|^{2} d s+3 t M_{F G} \int_{0}^{t} \mathbb{E}|x(\delta(s))|^{2} d s
\end{aligned}
$$

Because of $t<h \tau \leq \tau_{0}+\tau$ and $\mathbb{E}|x(\delta(s))|^{2} \geq 0$, we have

$$
\begin{aligned}
& \mathbb{E}|x(t)-x(\delta(t))|^{2} \\
\leq & 3\left[\left(\tau+\tau_{0}\right) M_{A}+M_{B}\right] \int_{n \tau-\tau_{0}}^{t} \mathbb{E}|x(s)|^{2} d s+3\left(\tau+\tau_{0}\right) M_{F G} \int_{n \tau-\tau_{0}}^{t} \mathbb{E}|x(\delta(s))|^{2} d s
\end{aligned}
$$

Someone may notice that $\int_{n \tau-\tau_{0}}^{t} \mathbb{E}|x(\delta(s))|^{2} d s$ implies an initial data $x_{\theta}:=\left\{x(\theta),-2 \tau_{0}-\tau \leq \theta \leq 0\right\}$, such for $n=0$. This is why we need to define $x(\theta)=y(0)=x\left(-\tau_{0}\right)$ for all $\theta \in\left[-2 \tau_{0}-\tau, \tau_{0}\right)$ in the end 
of Section 2.

$$
\begin{aligned}
& \mathbb{E}|x(t)-x(\delta(t))|^{2} \\
\leq & {\left[6\left(\tau+\tau_{0}\right) M_{A}+6 M_{B}\right] \int_{n \tau-\tau_{0}}^{t} \mathbb{E}|x(s)-x(\delta(s))|^{2} d s } \\
+ & {\left[6\left(\tau+\tau_{0}\right) M_{A}+6 M_{B}+3\left(\tau+\tau_{0}\right) M_{F G}\right] \int_{n \tau-\tau_{0}}^{t} \mathbb{E}|x(\delta(s))|^{2} d s }
\end{aligned}
$$

Thus we get the exact same property as (3.4). It is easy to see that (3.5) still works for $0 \leq t<h \tau$, so this property holds for any $t \geq 0$.

Now for any $t \geq 0$, we divide the interval $[0, t]$ into subintervals of length, at most, $\tau$ to get

$$
\int_{0}^{t} \mathbb{E}|x(s)-x(\delta(s))|^{2} d s=\int_{n \tau}^{t} \mathbb{E}|x(s)-x(\delta(s))|^{2} d s+\sum_{k=0}^{n-1} \int_{(n-k-1) \tau}^{(n-k) \tau} \mathbb{E}|x(s)-x(\delta(s))|^{2} d s .
$$

For each subinterval, applying (3.5) yields

$$
\begin{aligned}
\int_{0}^{t} \mathbb{E}|x(s)-x(\delta(s))|^{2} d s & \leq K\left(\tau, \tau_{0}\right) \int_{n \tau}^{t} \sum_{k=0}^{h} \mathbb{E}\left|x\left((n-k) \tau-\tau_{0}\right)\right|^{2} d s \\
& +K\left(\tau, \tau_{0}\right) \sum_{k=0}^{n-1} \int_{(n-k-1) \tau}^{(n-k) \tau} \sum_{l=n-h-1-k}^{n-1-k} \mathbb{E}\left|x\left(l \tau-\tau_{0}\right)\right|^{2} d s
\end{aligned}
$$

Then by the definition of the function $\delta(t)$, we have

$$
\begin{aligned}
\int_{0}^{t} \mathbb{E}|x(s)-x(\delta(s))|^{2} d s & \leq K\left(\tau, \tau_{0}\right) \sum_{k=0}^{h} \int_{(n-k) \tau}^{t-k \tau} \mathbb{E}|x(\delta(s))|^{2} d s \\
& +K\left(\tau, \tau_{0}\right) \sum_{k=0}^{n-1} \sum_{l=0}^{h} \int_{(n-1-k-l) \tau}^{(n-k-l) \tau} \mathbb{E}|x(\delta(s))|^{2} d s
\end{aligned}
$$

Combining the corresponding integrals, we get

$$
\begin{aligned}
& \int_{0}^{t} \mathbb{E}|x(s)-x(\delta(s))|^{2} d s \\
\leq & K\left(\tau, \tau_{0}\right) \sum_{k=0}^{h} \int_{-k \tau}^{t-k \tau} \mathbb{E}|x(\delta(s))|^{2} d s \\
\leq & (h+1) K\left(\tau, \tau_{0}\right) \int_{0}^{t} \mathbb{E}|x(\delta(s))|^{2} d s+K\left(\tau, \tau_{0}\right) \sum_{k=1}^{h} \int_{-k \tau}^{0} \mathbb{E}|x(\delta(s))|^{2} d s \\
\leq & (h+1) K\left(\tau, \tau_{0}\right) \int_{0}^{t} \mathbb{E}|x(\delta(s))|^{2} d s+\frac{\tau}{2}\left(h+h^{2}\right) K\left(\tau, \tau_{0}\right)_{\theta \in\left[-h \tau-\tau_{0}, 0\right]} \mathbb{E}|x(\theta)|^{2} \\
\leq & 2(h+1) K\left(\tau, \tau_{0}\right) \int_{0}^{t}\left(\mathbb{E}|x(s)-x(\delta(s))|^{2}+\mathbb{E}|x(s)|^{2}\right) d s \\
+ & \frac{\tau}{2}\left(h+h^{2}\right) K\left(\tau, \tau_{0}\right) \sup _{\theta \in\left[-h \tau-\tau_{0}, 0\right]} \mathbb{E}|x(\theta)|^{2} \\
\leq & \frac{2(h+1) K\left(\tau, \tau_{0}\right)}{1-2(h+1) K\left(\tau, \tau_{0}\right)} \int_{0}^{t} \mathbb{E}|x(s)|^{2} d s+\frac{\tau\left(h+h^{2}\right) K\left(\tau, \tau_{0}\right)}{2-4(h+1) K\left(\tau, \tau_{0}\right)} \underset{\theta \in\left[-h \tau-\tau_{0}, 0\right]}{\sup |x(\theta)|^{2} .} \mathbb{E}
\end{aligned}
$$

Since $\sup _{\theta \in\left[-h \tau-\tau_{0}, 0\right]} \mathbb{E}|x(\theta)|^{2}=\sup _{\theta \in\left[-\tau_{0}, 0\right]} \mathbb{E}|x(\theta)|^{2}$, the proof is complete. 
Theorem 3.2. Assume that there are symmetric positive-definite matrices $Q(i)=Q_{i}(i \in S)$ such that

$$
\bar{Q}(i)=\bar{Q}_{i}:=Q_{i}\left(A_{i}+F_{i} G_{i}\right)+\left(A_{i}+F_{i} G_{i}\right)^{T} Q_{i}+\sum_{k=1}^{m} B_{k i}^{T} Q_{i} B_{k i}+\sum_{j=1}^{N} \gamma_{i j} Q_{j}
$$

are all negative-definite matrices. Set

$$
-\lambda:=\max _{i \in S} \lambda_{\max }\left(\bar{Q}_{i}\right)<0, \quad M_{Q F G}=\max _{i \in S}\left\|Q_{i} F_{i} G_{i}\right\|^{2}
$$

If $\tau$ and $\tau_{0}$ are both sufficiently small for $\lambda>2 \lambda_{\tau, \tau_{0}}$, where

$$
\lambda_{\tau, \tau_{0}}=\sqrt{\frac{2 M_{Q F G}(h+1) K\left(\tau, \tau_{0}\right)}{1-2(h+1) K\left(\tau, \tau_{0}\right)}},
$$

then the solution of $S D D E(3.1)$ satisfies

$$
\mathbb{E}|x(t)|^{2} \leq\left(\frac{\lambda_{M}}{\lambda_{m}}+\frac{h \tau \lambda_{\tau, \tau_{0}}}{4 \lambda_{m}}\right)\left(\sup _{\theta \in\left[-\tau_{0}, 0\right]} \mathbb{E}|x(\theta)|^{2}\right) e^{\frac{-\left(\lambda-2 \lambda_{\tau, \tau_{0}}\right) t}{\lambda_{m}}}, \quad \forall t \geq 0,
$$

where $\lambda_{M}=\max _{i \in S} \lambda_{\max }\left(Q_{i}\right), \lambda_{m}=\min _{i \in S} \lambda_{\min }\left(Q_{i}\right), h$ and $K\left(\tau, \tau_{0}\right)$ have been defined in Lemma3.1. In other words, SDDE(3.1) is exponentially stable in mean square.

Proof. Applying the generalized Itô formula to $x^{T}(t) Q(r(t)) x(t)$ we can get

$$
\begin{aligned}
& d\left[x^{T}(t) Q(r(t)) x(t)\right] \\
= & {\left[x^{T}(t) \bar{Q}(r(t)) x(t)-2 x^{T}(t) Q(r(t)) F(r(t)) G(r(t))(x(t)-x(\delta(t)))\right] d t+d M_{1}(t), }
\end{aligned}
$$

where $M_{1}(t)$ is a martingales with $M_{1}(0)=0$. The form of $M_{1}(t)$ is not used, so it is not specified here. Integrating both sides from 0 to $t$ and taking expectation yields

$$
\begin{aligned}
& \lambda_{m} \mathbb{E}|x(t)|^{2} \\
\leq & \lambda_{M} \mathbb{E}\left|x_{0}\right|^{2}-\lambda \int_{0}^{t} \mathbb{E}|x(s)|^{2} d s+2 \sqrt{M_{Q F G}} \int_{0}^{t} \mathbb{E}(|x(s)||x(s)-x(\delta(s))|) d s \\
\leq & \lambda_{M} \mathbb{E}\left|x_{0}\right|^{2}-\lambda \int_{0}^{t} \mathbb{E}|x(s)|^{2} d s+\lambda_{\tau, \tau_{0}} \int_{0}^{t} \mathbb{E}|x(s)|^{2} d s+\frac{M_{Q F G}}{\lambda_{\tau, \tau_{0}}} \int_{0}^{t} \mathbb{E}|x(s)-x(\delta(s))|^{2} d s
\end{aligned}
$$

By Lemma 3.1, we have

$$
\begin{aligned}
& \lambda_{m} \mathbb{E}|x(t)|^{2} \\
\leq & \lambda_{M} \mathbb{E}\left|x_{0}\right|^{2}-\lambda \int_{0}^{t} \mathbb{E}|x(s)|^{2} d s+\lambda_{\tau, \tau_{0}} \int_{0}^{t} \mathbb{E}|x(s)|^{2} d s \\
+ & \frac{M_{Q F G}}{\lambda_{\tau, \tau_{0}}}\left[\frac{2(h+1) K\left(\tau, \tau_{0}\right)}{1-2(h+1) K\left(\tau, \tau_{0}\right)} \int_{0}^{t} \mathbb{E}|x(s)|^{2} d s+\frac{\tau\left(h+h^{2}\right) K\left(\tau, \tau_{0}\right)}{2-4(h+1) K\left(\tau, \tau_{0}\right)} \sup _{\theta \in\left[-\tau_{0}, 0\right]} \mathbb{E}\left|x_{0}\right|^{2}\right] \\
\leq & \lambda_{M} \mathbb{E}\left|x_{0}\right|^{2}-\lambda \int_{0}^{t} \mathbb{E}|x(s)|^{2} d s+\lambda_{\tau, \tau_{0}} \int_{0}^{t} \mathbb{E}|x(s)|^{2} d s \\
+ & \lambda_{\tau, \tau_{0}} \int_{0}^{t} \mathbb{E}|x(s)|^{2} d s+\frac{h \tau \lambda_{\tau, \tau_{0}}}{4} \sup _{\theta \in\left[-\tau_{0}, 0\right]} \mathbb{E}\left|x_{0}\right|^{2} \\
\leq & \left(\lambda_{M}+\frac{h \tau \lambda_{\tau, \tau_{0}}}{4} \sup _{\theta \in\left[-\tau_{0}, 0\right]} \mathbb{E}\left|x_{0}\right|^{2}-\left(\lambda-2 \lambda_{\tau, \tau_{0}}\right) \int_{0}^{t} \mathbb{E}|x(s)|^{2} d s .\right.
\end{aligned}
$$


The Gronwall inequality then indicates

$$
\mathbb{E}|x(t)|^{2} \leq\left(\frac{\lambda_{M}}{\lambda_{m}}+\frac{h \tau \lambda_{\tau, \tau_{0}}}{4 \lambda_{m}}\right)\left(\sup _{\theta \in\left[-\tau_{0}, 0\right]} \mathbb{E}|x(\theta)|^{2}\right) e^{\frac{-\left(\lambda-2 \lambda_{\tau, \tau_{0}}\right) t}{\lambda_{m}}} .
$$

The proof is complete.

\section{Main Result: Noninear Case}

In this section, we shall discuss a more general nonlinear problem that

$$
d x(t)=f(x(t), r(t), t) d t+g(x(t), r(t), t) d \omega(t)
$$

with the following assumptions.

assp4.1

Assumption 4.1. For each $i \in S$, there is a pair of symmetric $n \times n$-matrices $P_{i}$ and $\bar{P}_{i}$ with $P_{i}$ being positive-definite such that

$$
2 x^{T} P_{i} f(x, i, t)+g^{T}(x, i, t) P_{i} g(x, i, t) \leq x^{T} \bar{P}_{i} x
$$

for all $(x, i, t) \in \mathbb{R}^{n} \times S \times \mathbb{R}_{+}$.

assp4.2 Assumption 4.2. There is a pair of positive constants $\alpha_{1}$ and $\alpha_{2}$ such that

$$
|f(x, i, t)|^{2} \leq \alpha_{1}|x|^{2} \quad \text { and } \quad|g(x, i, t)|^{2} \leq \alpha_{2}|x|^{2}
$$

for all $x, i, t) \in \mathbb{R}^{n} \times S \times \mathbb{R}_{+}$.

Suppose that the system (4.1) is unstable and we are required to design a control function. Due to the linear growth conditions assumed on both $f$ and $g$, a linear time delay feedback control could be sufficient. We design the control function as $u(x(\delta(t)), r(t), t)=F(r(t)) G(r(t)) x(\delta(t))$ and the controlled system is then denoted as

$$
d x(t)=[f(x(t), r(t), t)+F(r(t)) G(r(t)) x(\delta(t))] d t+g(x(t), r(t), t) d \omega(t) .
$$

Now let us present a useful lemma similar to Lemma (3.1).

Lemma 4.3. Let Assumption(4.2) hold. Set

$$
\alpha_{3}=\max _{i \in S}\left\|F_{i} G_{i}\right\|^{2}
$$

and define

$$
H\left(\tau, \tau_{0}\right)=\tau\left[6\left(\tau+\tau_{0}\right) \alpha_{1}+6 \alpha_{2}+3\left(\tau+\tau_{0}\right) \alpha_{3}\right] e^{\left[6\left(\tau+\tau_{0}\right) \alpha_{1}+6 \alpha_{2}\right]\left(\tau+\tau_{0}\right)}
$$


for $\tau>0$ and $\tau_{0}>0$. If $\tau$ and $\tau_{0}$ are both sufficiently small for the $2(h+1) H\left(\tau, \tau_{0}\right)<1$, where $h$ is same as (3.1), then the solution $x(t)$ of SDDE (4.2) satisfied

$$
\begin{aligned}
& \int_{0}^{t} \mathbb{E}|x(s)-x(\delta(s))|^{2} d s \\
& \leq \frac{2(h+1) H\left(\tau, \tau_{0}\right)}{1-2(h+1) H\left(\tau, \tau_{0}\right)} \int_{0}^{t} \mathbb{E}|x(s)|^{2} d s+\frac{\tau\left(h+h^{2}\right) H\left(\tau, \tau_{0}\right)}{2-4(h+1) H\left(\tau, \tau_{0}\right)} \sup _{-\tau_{0} \leq \theta \leq 0} \mathbb{E}|x(\theta)|^{2} .
\end{aligned}
$$

for all $t \geq 0$.

This lemma can be proved in the same way as Lemma3.1 was proved so we omit the proof.

Theorem 4.4. Let Assumption4.1 and 4.2 hold. Assume that the following LMIs

$$
U_{i}:=\bar{P}_{i}+P_{i} F_{i} G_{i}+G_{i}^{T} F_{i}^{T} P_{i}+\sum_{j=1}^{N} \gamma_{i j} P_{j}<0, \quad i \in S
$$

have their solutions $F_{i}(i \in S)$ in the case of feedback control (i.e. $G_{i}$ 's are given) or their solutions $G_{i}$ in the case of output injection (i.e. $F_{i}$ 's are given). Set

$$
-\eta:=\max _{i \in S} \lambda_{\max }\left(U_{i}\right)<0 \quad \text { and } \quad \alpha_{4}=\max _{i \in S}\left\|P_{i} F_{i} G_{i}\right\|^{2} .
$$

If $\tau$ and $\tau_{0}$ are both sufficiently small for $\eta>2 \eta_{\tau, \tau_{0}}$, where

$$
\eta_{\tau, \tau_{0}}:=\sqrt{\frac{2 \alpha_{4}(h+1) H\left(\tau, \tau_{0}\right)}{1-2(h+1) H\left(\tau, \tau_{0}\right)}}
$$

then the solution of $S D D E(4.2)$ satisfies

$$
\mathbb{E}|x(t)|^{2} \leq\left(\frac{\eta_{M}}{\eta_{m}}+\frac{h \tau \eta_{\tau, \tau_{0}}}{4 \eta_{m}}\right)\left(\sup _{\theta \in\left[-\tau_{0}, 0\right]} \mathbb{E}|x(\theta)|^{2}\right) e^{\frac{-\left(\eta-2 \eta_{\left.\tau, \tau_{0}\right) t}\right.}{\eta_{m}}}, \quad \forall t \geq 0,
$$

where $\eta_{M}=\max _{i \in S} \lambda_{\max }\left(P_{i}\right), \eta_{m}=\min _{i \in S} \lambda_{\min }\left(P_{i}\right), H\left(\tau, \tau_{0}\right)$ has been defined in Lemma4.3. In other words, SDDE(4.2) is exponentially stable in mean square.

Proof. This theorem can be proved in a similar way as Theorem 3.2 was proved so we only give the key steps. Applying the generalized Itô formula to $x^{T}(t) P(r(t)) x(t)$ we get

$$
\begin{aligned}
& d\left[x^{T}(t) P(r(t)) x(t)\right] \\
& =\left[x^{T}(t) \bar{U}(r(t)) x(t)-2 x^{T}(t) P(r(t)) F(r(t)) G(r(t))(x(t)-x(\delta(t)))\right] d t+d M_{3}(t) .
\end{aligned}
$$

where $M_{3}(t)$ is a martingale with $M_{3}(0)=0$. Then we have

$$
\begin{aligned}
& \eta_{m} \mathbb{E}|x(t)|^{2} \\
& \leq \eta_{M} \mathbb{E}\left|x_{0}\right|^{2}-\eta \int_{0}^{t} \mathbb{E}|x(s)|^{2} d s+\eta_{\tau, \tau_{0}} \int_{0}^{t} \mathbb{E}|x(s)|^{2} d s+\frac{\alpha_{4}}{\eta_{\tau, \tau_{0}}} \int_{0}^{t} \mathbb{E}|x(s)-x(\delta(s))|^{2} d s
\end{aligned}
$$

By Lemma(4.3), we can then obtain

$$
\eta_{m} \mathbb{E}|x(t)|^{2} \leq\left(\eta_{M}+\frac{h \tau \eta_{\tau, \tau_{0}}}{4}\right)\left(\sup _{\theta \in\left[-\tau_{0}, 0\right]} \mathbb{E}|x(\theta)|^{2}\right)-\left(\eta-2 \eta_{\tau, \tau_{0}}\right) \int_{0}^{t} \mathbb{E}|x(s)|^{2} d s
$$

Which yields (4.7). The proof is complete. 


\section{Design of the Control Function and Numerical Example}

In this section, we shall brief the design of the control function then discuss an example to illustrate the theory.

The method of linear matrix inequalities (LMIs) [24, 28] is one of the commonly used technique in the study of stability of linear systems. Although there are other methods (see the survey paper [29]), we shall adopt the LMIs in this paper.

We only demonstrate the case of state feedback control here. The similar idea works for the output injection control. Given $G(\cdot)$ is known, to apply Theorem 3.2 we need to find $F_{i}(i \in S)$ and positivedefinite symmetric matrices $Q_{i}(i \in S)$ to solve the following matrix inequalities

$$
Q_{i}\left(A_{i}+F_{i} G_{i}\right)+\left(A_{i}+F_{i} G_{i}\right)^{T} Q_{i}+\sum_{k=1}^{m} B_{k i}^{T} Q_{i} B_{k i}+\sum_{j=1}^{N} \gamma_{i j} Q_{j}<0, \quad i \in S .
$$

The inequality system above is not linear in $Q_{i}$ and $F_{i}$. But by setting $Y_{i}=Q_{i} F_{i}$, we see the inequality system becomes

$$
Q_{i} A_{i}+Y_{i} G_{i}+A_{i}^{T} Q_{i}+G_{i}^{T} Y_{i}^{T}+\sum_{k=1}^{m} B_{k i}^{T} Q_{i} B_{k i}+\sum_{j=1}^{N} \gamma_{i j} Q_{j}<0, \quad i \in S .
$$

If the above LMIs have their solutions $Q_{i}=Q_{i}^{T}>0$ and $Y_{i}(i \in S)$, then setting $F_{i}=Q_{i}^{-1} Y_{i}$ we have (5.1). Choosing $\tau$ and $\tau_{0}$ to be sufficiently small such that $\lambda>\lambda_{\tau, \tau_{0}}$, Theorem 3.2 indicates the system (3) is mean-square exponentially stable.

For the nonlinear system in Section 4, two steps are needed to apply Theorem 4.4:

1. we need to look for the $2 N$ matrices $P_{i}$ and $\bar{P}_{i}$ for Assumption 4.1 to hold;

2. we need to solve the LMIs in (4.5) for their solutions $F_{i}$ (or $G_{i}$ ).

There are some available computer software, such as Matlab, for step 2, and the ideas for step 1 have been discussed in [26]. So we omit it here.

Now we give an simulation example.

Example 5.1. Let us first consider an 2-dimensional hybrid system,

$$
d x(t)=A(r(t)) x(t) d t+B(r(t)) x(t) d \omega(t)
$$

on $t \geq 0$. Here $\omega(t)$ is a scalar Brownian motion; $r(t)$ is a Markov chain on the state space $S=1,2$ with the generator

$$
\Gamma=\left[\begin{array}{rr}
-1 & 1 \\
1 & -1
\end{array}\right]
$$


and the system matrices are

$$
\begin{array}{cc}
A_{1}=\left[\begin{array}{rr}
1 & 3 \\
4 & -5
\end{array}\right], & A_{2}=\left[\begin{array}{rr}
-3 & 4 \\
5 & 2
\end{array}\right], \\
B_{1}=\left[\begin{array}{ll}
1 & 2 \\
1 & 0
\end{array}\right], & B_{2}=\left[\begin{array}{ll}
1 & 1 \\
1 & 1
\end{array}\right] .
\end{array}
$$

The computer simulation (Figure 1) shows that this hybrid SDE is not mean-square exponentially stable.(The simulation of the path illustrate that it is not almost surely exponential stable thus not mean-square exponential stable [1].)
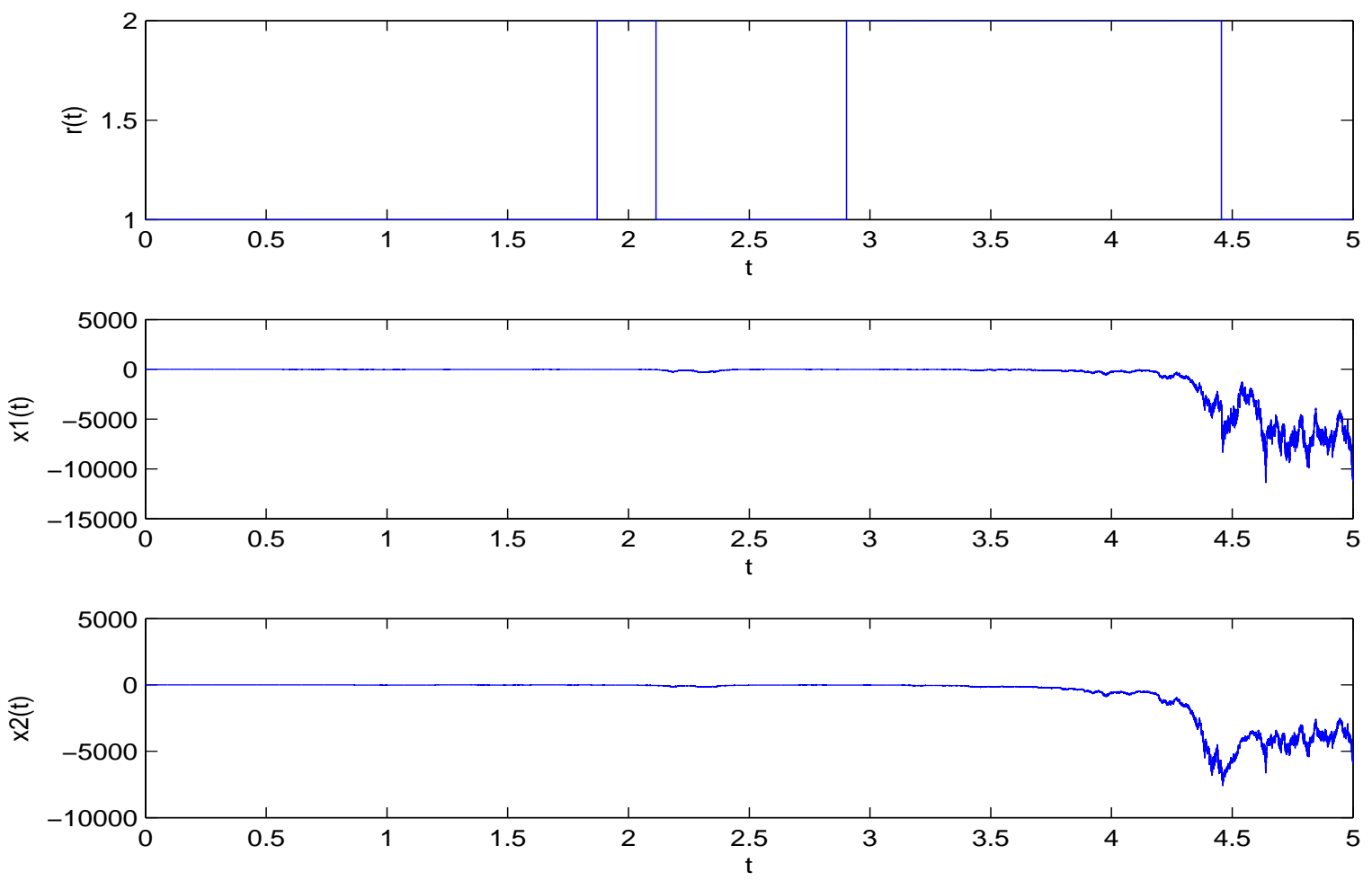

Figure 1: Computer simulation of the paths of $r(t), x_{1}(t)$ and $x_{2}(t)$ for the hybrid $\operatorname{SDE}(5.2)$

using the Euler-Maruyama method with step size $10^{-6}$ and initial values $r(0)=1, x_{1}(0)=-2$ and

$$
x_{2}(0)=1 \text {. }
$$

Assume that we can observe the state with a time delay $\tau_{0}$, which means we can observe the state $x\left(t-\tau_{0}\right)$ at time $t$. In this situation, let us now design a discrete-time state feedback control to stabilize the system with sampling gap $\tau$. Assume that the controlled system has the following form

$$
d x(t)=[A(r(t)) x(t)+F(r(t)) G(r(t)) x(\delta(t))] d t+B(r(t)) x(t) d \omega(t)
$$

where $G_{1}=(1,1)$ and $G_{2}=(1,1)$. Our aim is to seek for $F_{1}$ and $F_{2}$ in $R^{2 \times 1}$ and then find the condition $\tau$ fitted so that the controlled system to be exponentially stable in mean square. According to Theorem(3.2), we find the $\operatorname{LMIs}(5.1)$ have the following set of solutions $Q_{1}=Q_{2}=I$ (the $2 \times 2$ identity 
matrix) and

$$
F_{1}=\left[\begin{array}{l}
-7 \\
-2
\end{array}\right], \quad F_{2}=\left[\begin{array}{l}
-3 \\
-8
\end{array}\right]
$$

and for these solutions we have

$$
\overline{Q_{1}}=\left[\begin{array}{rr}
-10 & 0 \\
0 & -10
\end{array}\right], \quad \bar{Q}_{2}=\left[\begin{array}{rr}
-10 & 0 \\
0 & -10
\end{array}\right] \text {. }
$$

It is easy to compute the parameters, we have

$$
\lambda=10, \quad M_{A}=44.51, \quad M_{B}=5.24, \quad M_{F G}=146 .
$$

Through our calculation, we have the exponential stability whenever $(h+1) K\left(\tau, \tau_{0}\right)<25 / 342$, where

$$
K\left(\tau, \tau_{0}\right)=\tau\left[705.06\left(\tau+\tau_{0}\right)+31.44\right] e^{267.06\left(\tau+\tau_{0}\right)^{2}+31.44\left(\tau+\tau_{0}\right)} .
$$

For example, if the time delay $\tau_{0}=0.001$, then we need $\tau \leq 0.001$, if the time delay $\tau_{0}=0.0012$, then we need $\tau \leq 0.0007$ to make the controlled system(5.3) to be exponential stable in mean square. The computer simulation (Figure 2) supports this result clearly.
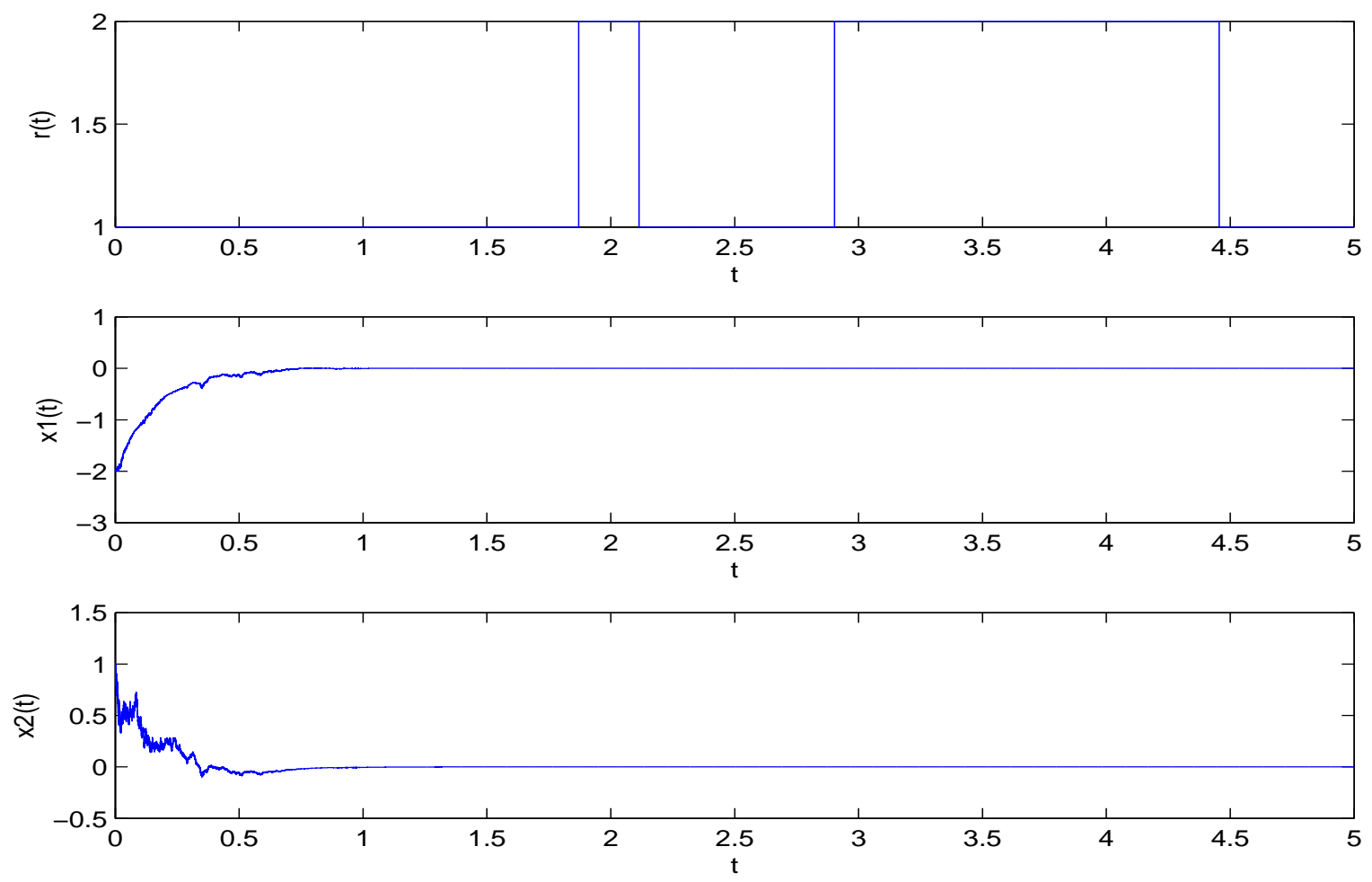

Figure 2: Computer simulation of the paths of $r(t), x_{1}(t)$ and $x_{2}(t)$ for the hybrid $\operatorname{SDDE}(5.3)$ with $\tau_{0}=0.001$ and $\tau=0.001$ using the Euler-Maruyama method with step size $10^{-6}$ and initial values $r(0)=1, x_{1}(0)=-2$ and $x_{2}(0)=1$. 


\section{Conclusions and Future Research}

This paper studied the stabilisation of SDEs with Markovian switching by the feedback control based on discrete-time state observation with a time delay. With the time delay taken into consideration, the control function becomes more realistic. We only considered the mean-square stability in this article, but other stability properties, such as almost sure stability, are also interesting. This could be one of the future research.

[1] X. Mao, C. Yuan, Stochastic Differential Equations with Markovian Switching, Imperial College Press, London, 2006.

[2] G. Yin, C. Zhu, Hybrid Switching Diffusions: Properties and Applications, in: Stochastic Modelling and Applied Probability, vol. 63, Springer, New York, 2010.

[3] Q. Liu, Asymptotic properties of a stochastic n-species Gilpin-Ayala competitive model with Lvy jumps and Markovian switching, Commun. Nonlinear Sci. Numer. Simul. 26 (2015), no. 1-3, 1C10.

[4] G. Hu, Invariant distribution of stochastic Gompertz equation under regime switching, Math. Comput. Simulation 97 (2014), 192C206.

[5] M. Liu, K. Wang, Dynamics and simulations of a logistic model with impulsive perturbations in a random environment Math. Comput. Simulation 92 (2013), 53C75.

[6] J. Lv, K. Wang, X. Zou, Remarks on stochastic permanence of population models, J. Math. Anal. Appl. 408 (2013), no. 2, 561C571.

[7] Allwright, J.C., Astolfi, A. and Wong, H.P., A note on asymptotic stabilization of linear systems by periodic, piecewise constant, output feedback, Automatica 41(2) (2005), 339-344.

[8] Basak, G.K., Bisi, A. and Ghosh, M.K., Stability of a random diffusion with linear drift, J. Math. Anal. Appl. 202 (1996), 604-622.

[9] Ebihara, Y., Yamaguchi, J. and Hagiwara, T., Periodically time-varying controller synthesis for multiobjective $\mathrm{H}_{2} / \mathrm{H}_{\infty}$ control of discrete-time systems and analysis of achievable performance, Systems Control Lett. 60(9) (2011), 709-717.

[10] Chammas, A.B. and Leondes, C.T., On the finite time control of linear systems by piecewise constant output feedback, Internat. J. Control 30(2) (1979), 227-234.

[11] Hagiwara,T. and Araki, M., Design of stable state feedback controller based on the multirate sampling of the plant output, IEEE Trans. Automat. Control 33(9) (1988), 812-819.

[12] Ji, Y. and Chizeck, H.J., Controllability, stabilizability and continuous-time Markovian jump linear quadratic control, IEEE Trans. Automat. Control 35 (1990), 777-788. 
M99 [13] Mao, X., Stability of stochastic differential equations with Markovian switching, Sto. Proc. Their Appl. 79 (1999), 45-67.

M02 [14] Mao, X., Exponential stability of stochastic delay interval systems with Markovian switching, IEEE Trans. Auto. Control 47(10) (2002), 1604-1612.

M13 [15] Mao,X., Stabilization of continuous-time hybrid stochastic differential equations by discrete-time feedback control, Automatica 49(12) (2013), 3677-3681.

MMP [16] Mao, X., Matasov, A. and Piunovskiy, A.B., Stochastic differential delay equations with Markovian switching, Bernoulli 6(1) (2000), 73-90.

MYY [17] Mao, X., Yin, G. and Yuan, C., Stabilization and destabilization of hybrid systems of stochastic differential equations, Automatica 43 (2007), 264-273.

Mar [18] Mariton, M., Jump Linear Systems in Automatic Control, Marcel Dekker, 1990.

Sha [19] Shaikhet, L., Stability of stochastic hereditary systems with Markov switching, Theory of Stochastic Processes 2(18) (1996), 180-184.

SMYI

[20] Shi, P., Mahmoud, M.S., Yi, J. and Ismail, A., Worst case control of uncertain jumping systems with multi-state and input delay information, Information Sciences 176 (2006), 186-200.

SLXZ

[21] Sun, M., Lam, J., Xu, S. and Zou, Y., Robust exponential stabilization for Markovian jump systems with mode-dependent input delay, Automatica 43 (2007), 1799-1807.

[22] Wei, G., Wang, Z., Shu, H. and Fang, J., Robust $H_{\infty}$ control of stochastic time-delay jumping systems with nonlinear disturbances, Optim. Control Appl. Meth. 27 (2006), 255-271.

YH [23] Yue, D. and Han, Q., Delay-dependent exponential stability of stochastic systems with time-varying delay, nonlinearity, and Markovian switching, IEEE Trans. Automat. Control 50 (2005), 217-222.

[24] X. Mao, J. Lam, L. Huang, Stabilisation of hybrid stochastic differential equations by delay feedback control, Systems Control Lett. 57 (2008), 927-935.

[25] X. Mao, W. Liu, L. Hu, Q. Luo, J. Lu, Stabilization of hybrid stochastic differential equations by feedback control based on discrete-time state observations, Systems Control Lett. 73 (2014), 88-95.

[26] S. You, W. Liu, J. Lu, X. Mao, Q. Qiu Stabilization of hybrid systems by feedback control based on discrete-time state observations, SIAM J. Control Optim. 53 (2015), no. 2, 905-925.

[27] Xu, S., Lam, J., Wang J.L. and Yang, G.H., Stabilization and $H_{\infty}$ control for uncertain stochastic time-delay systems via non-fragile controllers, Asian Journal of Control 8 (2006), 197-200.

M07 [28] Mao, X., Stability and stabilization of stochastic differential delay equations, IET Control Theory 6 Applications 1(6) (2007), 1551-1566. 